\title{
Plant cell culture combined with chemistry-a powerful route to complex natural products
}

\author{
James P. Kutney \\ Department of Chemistry, University of British Columbia, 2036 Main Mall, \\ Vancouver, B.C., Canada V6T $1 Z 1$
}

\begin{abstract}
The combination of plant cell culture methodology with synthetic chemistry affords an attractive route to the synthesis of complex natural products and related compounds of industrial importance. Plant cell cultures which often provide "unique" enzyme systems can be employed effectively as "reagents" in the biotransformation of synthetic substrates to desired end products. Such conversions are often superior to those utilizing chemical reagents with the result that the overall synthetic route is more efficient. Alternatively, the use of plant cell culture derived enzymes in the evaluation of biosynthetic pathways can often afford important information for synthetic design. The overall strategy illustrating such an approach is presented herein with specific examples.
\end{abstract}

\section{Introduction}

The plant kingdom has provided a wide variety of natural products with diverse chemical structures and a vast array of biological activities, many of which have found applications in the health sciences. For years, synthetic chemists have been afforded the challenge of developing syntheses of such components but often due to structural complexity the resulting multi-step syntheses rarely find application in large scale production as required in the pharmaceutical drug industry. As a result, starting materials for such drug production, or indeed the final clinical drug, are frequently obtained from tedious and often costly extraction from the living plant. This latter solution is often fraught with the well known problems: a) active agent present in minute amounts in the plant extract; b) separation of target compound may be difficult and, in turn, expensive; c) varying concentrations of the target compound depending on seasons during which plant collection is performed; d) desired plant species growing in geographically or politically inaccessible regions, etc.

Appropriate solutions to at least some of the above difficulties are possible by the use of plant cell culture methodology particularly when such studies are coupled with chemical methods. The advantages of plant cell cultures over living plants, in terms of secondary metabolite production, are clear: a) growth conditions are laboratory controlled, therefore, reproducible yields of end product are achieved; b) growth parameters such as $\mathrm{pH}$, changes in nutrient media, temperature, etc. can be optimized to achieve metabolite production in yields significantly higher than in the living plant; c) separation of target compounds is much easier due to lower complexity of extract; d) cloning of cell lines provides further optimization for end product yields; e) plant cell cultures are an excellent source of enzymes, much superior to living plants where isolation often leads to enzyme denaturation. With enzyme availability, the opportunity to perform biosynthetic and/or biotransformation experiments related to metabolite production, is clear.

It must be emphasized at the outset that the effectiveness of such a program depends entirely on an interdisciplinary "team" of scientists comprised of the "culture development" group (biologists, biochemists) and the "chemical" group, the latter isolating and characterizing the metabolites via spectroscopic methods coupled with synthesis as required. 
The present discussion will summarize studies performed in the author's laboratories, in several select areas, in order to portray to the potential reader the various avenues of research that such an interdisciplinary program can achieve.

\section{Avenues of Research}

Our program can be conveniently divided into four sections and the discussion following will present studies within these areas.

Section 1. Biosynthetic Information Forms a Basis for Efficient Synthesis of Complex

Natural Products.

Section 2. Plant Cell Cultures as "Reagents" in Organic Syntheses.

Section 3. Use of Plant Cell Cultures to Produce Higher Levels of Pharmaceutically Interesting Compounds.

Section 4. Use of Plant Cell Cultures to Separate Pharmacological Activities in Complex Herbal Medicine Extracts.

\section{Section 1. Biosynthetic Information Forms a Basis for Efficient Synthesis of Complex Natural} Products.

A large number of investigators have employed plant cell cultures with considerable advantage over living plants, for unravelling biosynthetic pathways of a variety of natural products. A stable cell line, once developed, can afford a reliable source of enzymes, the latter obtained under established conditions, to provide the opportunity for controlled experiments designed to obtain biosynthetic intermediates in a selected pathway. One of the particular interests in our program was to utilize information derived from such biosynthetic experiments to develop highly efficient syntheses of natural products. In the initial stages, we directed our attention to the clinically important anti-cancer drugs, vinblastine (VLB, 7) and vincristine (VCR, 7, replace Me by $\mathrm{CHO}$ ) - a problem that has attracted the interest of synthetic chemists for many years.

\subsection{Studies with the Catharanthus roseus Cell Line}

Detailed accounts of our extensive studies in this area involving development of cell lines, indole alkaloid production, isolation and immobilization of enzymes derived from the $C$. roseus cell line, are published. These experiments which also include biotransformation of "precursors" to unravel the probable structures of biosynthetic intermediates involved in the biosynthesis of VLB, form the subject of approximately 20 publications and of several recent summaries (1-3). For this reason, only a brief description of the most salient features to illustrate the approach of Section 1 will be presented here.

The isolated enzymes obtained from the $C$. roseus cell line (Fig. 1) were utilized in biosynthetic experiments with the alkaloids catharanthine (1) and vindoline (3) as "precursors" to initially establish the unstable dihydropyridinium intermediate (4) (Fig. 2) as the first formed biosynthetic intermediate.

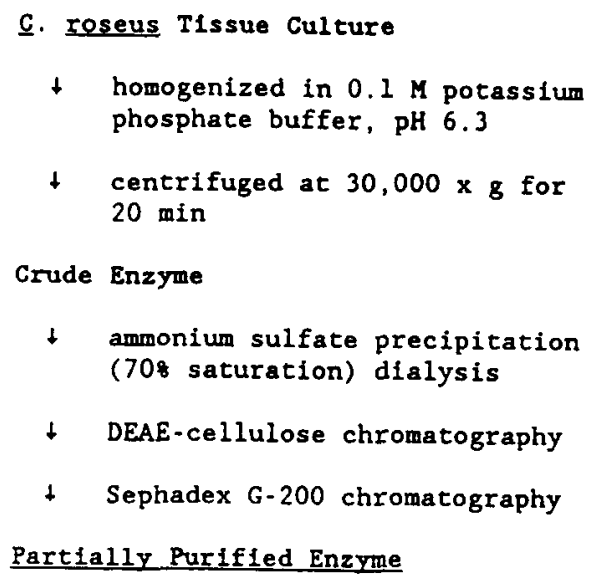

Fig. 1. Isolation of enzymes from cell culture of $C$. roseus. 


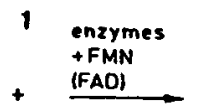

3
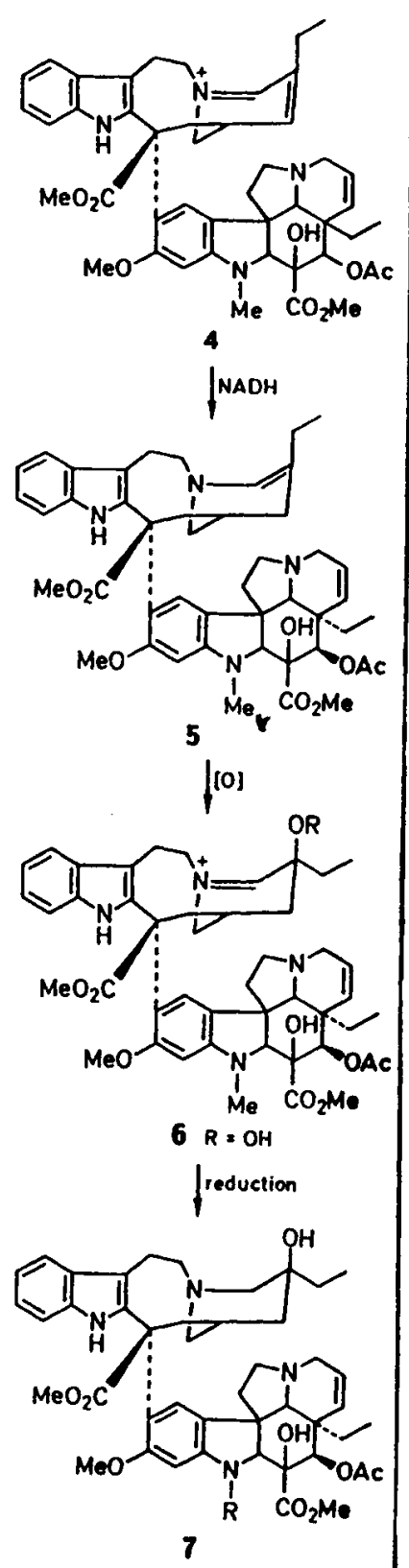

Fig. 2. Overall summary of the biosynthetic pathway of vinblastine ( 7 from catharanthine (1) and vindoline (3).

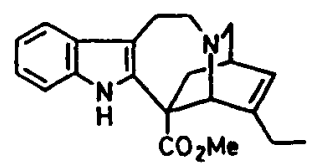

1

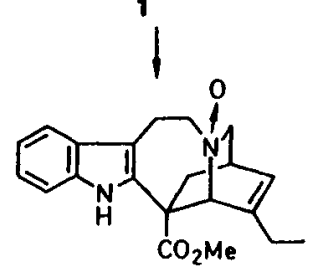

2

$+$

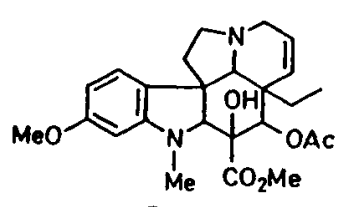

3

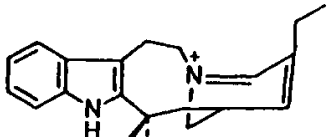

H

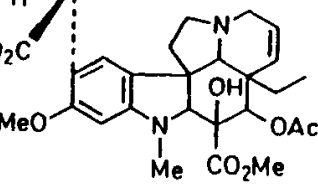

4
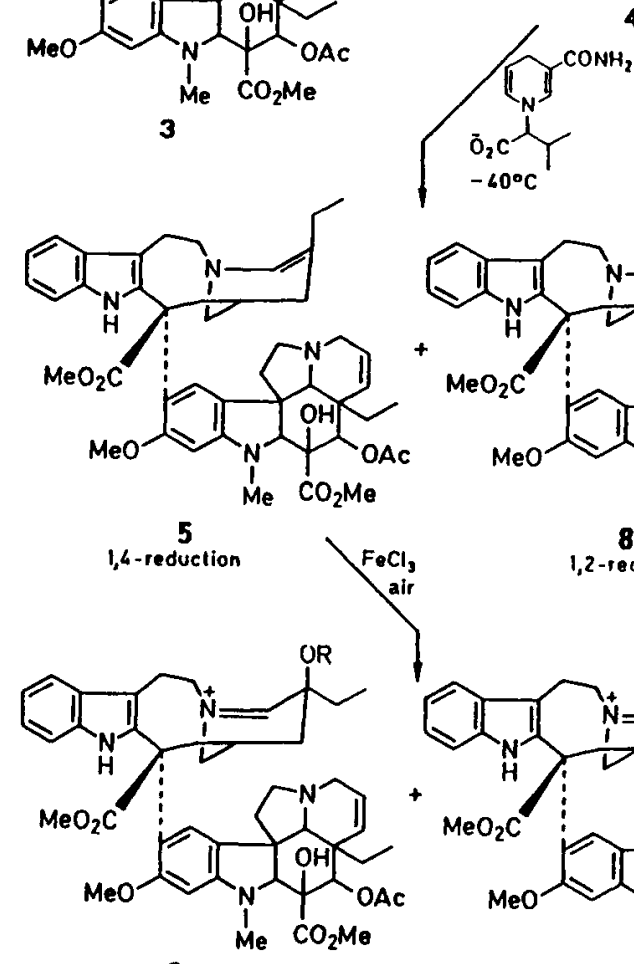

$6 \mathrm{R}=\mathrm{OH}$

$\mathrm{NaBH}_{4}$
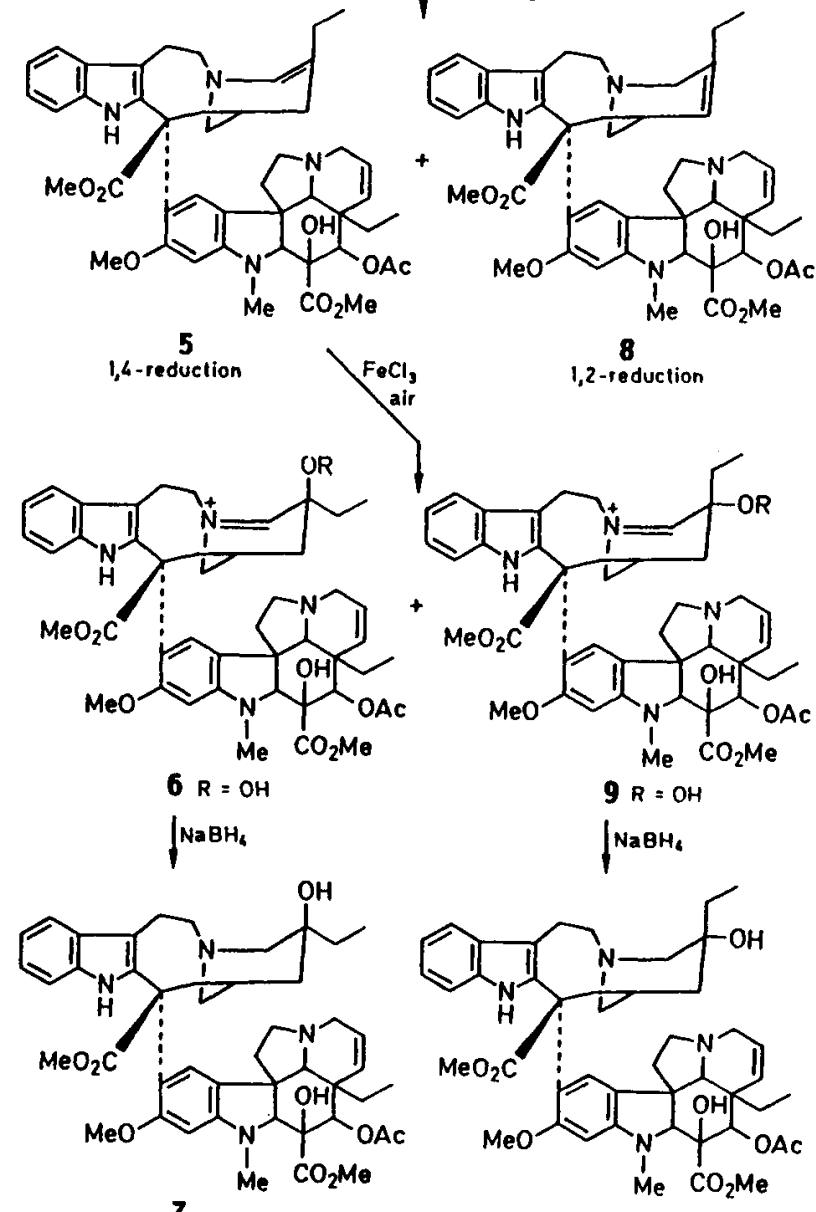

1,2 -reduction

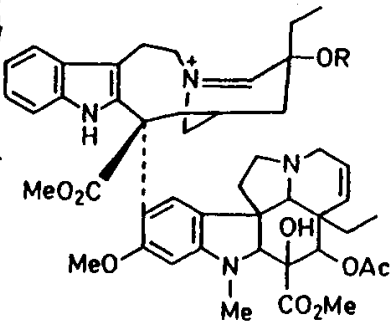

$9 \mathrm{R}=\mathrm{OH}$

$\mathrm{NaBH}_{6}$

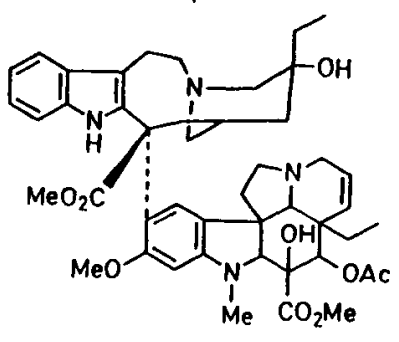

10

Fig. 3. A highly efficient "one-pot" process for the synthesis of vinblastine (7) and leurosidine (10) from catharanthine (1) and vindoline (3). 
With an enzyme preparation immobilized, via the technique of affinity gel chromatography, on $2^{\prime}, 5^{\prime}-$ ADP sepharose $(2,4)$, a 90\% "coupling" of 1 and 3 to 4 could be achieved in 15-30 min incubations. The enzyme(s) involved in this coupling process were shown to be of the "peroxidase" family. Longer incubation times (30-45 $\mathrm{min}$ ) led to the disappearance of 4 (HPLC monitoring) and the appearance of enamine 5. A separate experiment involving reduction of 4 to 5 by a commercial preparation of NADH established that such a reductive process involves NADH-like enzymes present in the enzyme preparation obtained via the isolation procedure shown in Fig. 1. Further incubation studies with the crude enzyme preparation revealed the disappearance of 5 to afford a new intermediate, proposed as 6 , and the latter finally converts to VLB (7). In summary, the sequence from $1+3 \rightarrow 4 \rightarrow 5 \rightarrow 6 \rightarrow 7$, involves oxidation, reduction, oxidation, reduction, in successive fashion. These data suggest that the biosynthesis of these bisindole alkaloids within the plant is likely a result of careful enzymatic control involving oxidative and reductive processes.

\subsection{Biomimetic Studies. Biosynthetic Information Sets the Stage for an Efficient Synthesis of Vinblastine.}

The overall enzymatic conversions summarized in Fig. 2 could now form the basis of a chemical synthetic route to VLB. Earlier studies in our laboratory (5) and elsewhere (6) had established that the chemical coupling of catharanthine (1) and vindoline (3) to 4 , could be achieved via catharanthine $\mathrm{N}$ oxide (2, Fig. 3), the latter being produced by MCPBA reaction with 1 . Coupling of 2 with 3 is successful with trifluoroacetic anhydride as a catalyst to stimulate a Polonovski-type reaction to afford 4. In effect, MCPBA becomes the chemical "mimic" for the peroxidase enzyme involved in the enzymecatalyzed coupling of 1 and 3 as shown in Fig. 2. We have established that the $\mathrm{N}$-oxide (2) is not accepted as a precursor in the enzymatic process to 4 so a different "activation" of 1 by the enzyme is involved (see discussion in ref. 1, 2 for details). The major focus in further studies directed to VLB was an efficient regiospecific 1,4-reduction of $\mathbf{4}$ to enamine 5 - a process which had not been achieved in our earlier studies or in other laboratories. The important information derived from the biosynthetic experiments was the realization that, instead of utilizing the more conventional reducing agents $\left(\mathrm{NaBH}_{4}\right.$, etc), which afforded only 1,2-reduction of 4 to anhydrovinblastine $(8)(5,6)$, chemical models mimicking the NADH enzyme-catalyzed process of 4 to 5 may become the reagents of choice. Indeed, an extensive study (7) with various dihydronicotinamides as reducing agents revealed an effective solution to this problem, as summarized in Fig. 3. After study with various oxidants $\left(\mathrm{O}_{2}, \mathrm{H}_{2} \mathrm{O}_{2}\right.$, $\mathrm{FeCl}_{3}$ ), the chemical parallel to the enzymatic oxidation of 5 to 6 was best achieved with $\mathrm{FeCl}_{3}$ and the final reduction, $6 \rightarrow 7$ was highly successful with $\mathrm{NaBH}_{4}$. The final objective, the direct conversion of 1 and 3 to vinblastine (7), and, in turn, vincristine (7, replace Me by $\mathrm{CHO}$ ) without isolation of intermediates, that is, a "one-pot" process could be realized. The overall process, involving five separate chemical reactions, and providing a $40 \%$ overall yield of vinblastine, demands that each reaction must proceed with yields in excess of $80 \%$. When the two additional bisindole products, anhydrovinblastine $(\mathbf{8}, 18 \%)$ and leurosidine $(10,17 \%)$ formed in this process are taken into account, it is clear that the majority of the reactions proceed in almost quantitative yield.

In conclusion, the studies with plant cell cultures of $C$. roseus have provided biosynthetic information which has formed the basis on which the development of an efficient synthetic route to a complex natural product has been achieved. The patented process is presently being prepared for commercial production of VLB and VCR.

\section{Section 2. Plant Cell Cultures as "Reagents" in Organic Synthesis.}

Frequent criticism levelled against the use of plant cell culture methods for production of secondary metabolites relates to the normally long periods (generally 2-3 weeks) associated with the synthesis of such natural products if production is desired directly within the nutrient media utilized for cell growth and/or propagation. An approach which alleviates this problem concerns the use of developed cell culture lines as "reagents" wherein the enzymes present within the culture, or isolated from the culture, can serve to biotransform "precursors" to suitable end products. Such enzymecatalyzed processes should generally complete in short time periods (minutes - hours) and should provide respectable yields of desired end products. An extensive program in our laboratory with well developed cell lines derived from the plant Catharanthus roseus (8), Podophyllum peltatum (9), Tripterygium wilfordii $(10)$, and Nicotiana sylvestris $(11,12)$, has revealed that the enzyme systems 


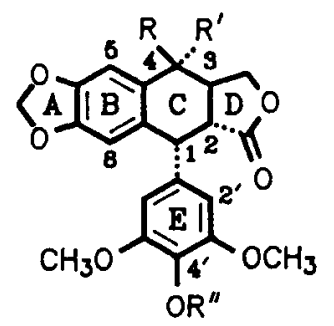

11

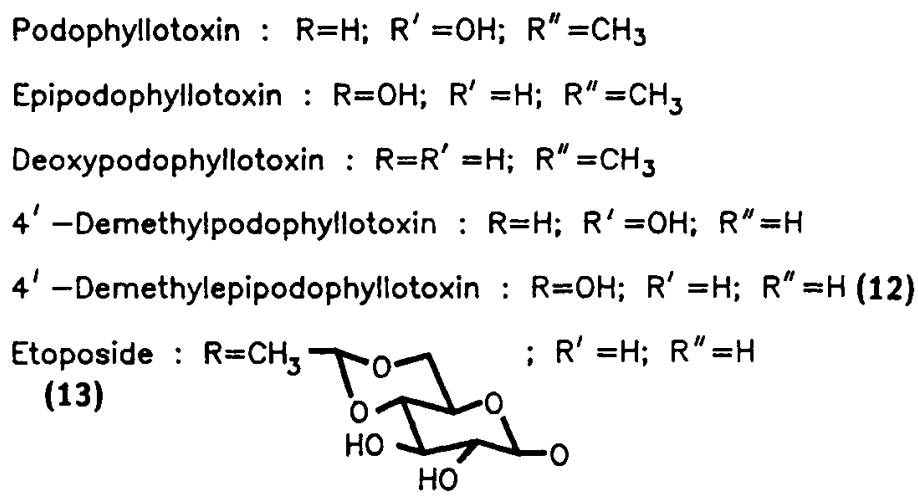

Fig. 4. The podophyllotoxin family of compounds.

present in such cell cultures do indeed possess considerable versatility. Such enzymes are capable of biotransforming "foreign" precursors, that is, substrates not normally produced in the plants from which the cultures are derived, to novel end products. It is appropriate to illustrate this approach with one example taken from our overall program.

\subsection{Studies with the Podophyllum peltatum cell line}

The podophyllotoxin family (11, Fig. 4) has been well studied over the years. Excellent reviews of the investigations pertaining to their chemistry and synthesis are available $(13,14)$ and the studies concerning their pharmacological properties, particularly the clinical importance of the anti-cancer drug etoposide (13) have been also reviewed $(13,15,16)$. The present route to this drug requires the isolation of podophyllotoxin, via extraction of Podophyllum peltatum plants, and subsequent utilization of this natural product as a starting material to afford etoposide. In brief, podophyllotoxin is converted, via a two step chemical process, to 4'-demethylepipodophyllotoxin (12, Fig. 4) and the latter, via a five step process to attach the carbohydrate unit, finally completes the commercial synthesis of this important drug.

In our program concerning the combined utilization of plant cell cultures and synthetic chemistry, we focussed our attention on deriving an efficient route to 12 . Such a strategy would eliminate the dependence on plant extraction and hopefully, some chemical conversions, to afford a more direct synthesis of 12 . If the subsequent attachment of the carbohydrate unit to 12 , so as to obtain the clinical drug, could be similarly improved through biotechnological methods, to be studied later, a highly attractive sequence to etoposide would be on hand. In order to ascertain which synthetic "precursors" may be suitable for biotransformation to 12 with the "reagent" enzymes present in the $P$. peltatum cell line, an understanding of the pertinent features of a proposed biosynthetic pathway leading to the podophyllotoxins is desirable. Dewick and coworkers (17) have published detailed studies in this area and have suggested that dibenzylbutanolides could act as substrates in enzyme-catalyzed oxidative cyclization reactions to the corresponding podophyllotoxins. Since our $P$. peltatum cell line had already provided several podophyllotoxins as metabolites (18), it was reasonable to assume that such substrates may be appropriate for the enzyme-catalyzed cyclizations with this cell line.

A requirement for this study involved the development of a versatile synthetic route to the requisite dibenzylbutanolides, for example, from commercially available starting materials. Indeed, such 
a route from the readily available aromatic aldehydes was completed (9) and studies with these substrates were undertaken.

2.1.1 Studies with whole cell fermentations - Semi-continuous process provides a "biological factory".

With a successful cell culture of $P$. peltatum which afforded podophyllotoxin, deoxypodophyllotoxin and 4'-demethylepipodophyllotoxin (Fig. 4) on hand (18), it was appropriate to evaluate the enzyme-catalyzed cyclization of the above-noted synthetic substrates. For the sake of brevity, one particular study involving the butanolide 14 and a semi-continuous process will be presented. In this approach, the fermentation broth, after a certain incubation period with 14, would be withdrawn under aseptic conditions, new medium, and a new batch of 14 added, and the process repeated over a number of cycles. Obviously, if successful, one batch of cell culture could be utilized for successive productions of $\mathbf{1 5}$ (Fig. 5), and this "biological factory" could afford a highly attractive approach toward multigram quantities of end products. Indeed, a successful approach has been developed in our laboratory ( 9 ) and only a brief description of a large number of experiments is provided here.<smiles>COc1cc([C@@H](O)[C@@H]2COC(=O)[C@H]2Cc2cc(O)c(O)c(OC)c2)ccc1OC(C)C</smiles>

14

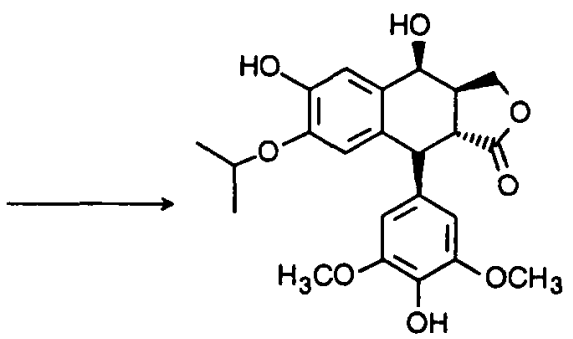

15

Fig. 5. Biotransformation of 14 to 15 with plant cell culture of $P$. peltatum (semi-continuous process) or with broth from cell culture of Nicotiana sylvestris.

Incubation of $\mathbf{1 4}$ in a Microferm bioreactor maintained according to the published procedure (18) with cells of varying ages (2-17 days old), was performed for $24-50 \mathrm{~h}$ periods. In a typical experiment, involving $24 \mathrm{~h}$ incubation periods between cycles, the fermentation broth containing the metabolites is withdrawn, new medium (generally at one-tenth to one-half original MS medium) and a new batch of 14 are added, and the process continued for a further $24 \mathrm{~h}$, whereupon the extraction, etc. is repeated. Although studies are still underway, we have already demonstrated that one batch of cells maintains the necessary enzymatic activity to biotransform 14 to 15 for several months, during which time 16 batches of 14 had been added and biotransformed to 15 . Indeed, we have not yet evaluated how long such activity persists since the supply of $\mathbf{1 4}$ was depleted, after numerous additions, and the study had to be terminated before enzymatic activity within the bioreactor had been exhausted. In effect, the $P$. peltatum cell line is a "biological factory" in terms of its ability to achieve oxidative coupling of dibenzylbutanolides to podophyllotoxin analogues. The present yields of $\mathbf{1 5}$ are in the range of $50 \%$ when 14 is added in $24-48 \mathrm{~h}$ cycles although higher yields are anticipated when conditions are optimized.

In summary, the semi-continuous process, once optimized, will afford a highly attractive route to various analogues of the podophyllotoxin series.

Finally, it should be noted that an efficient process in removing the isopropyl group in $\mathbf{1 5}$ and regeneration of the methylenedioxy function as required in 12 (Fig. 4) has already been completed in our laboratory.

\subsection{Studies with the Nicotiana sylvestris cell line.}

While the above studies were underway, we developed a stable cell line of $N$. sylvestris $(11,12)$ and were able to demonstrate, via the appropriate enzyme assay, that this cell line produces high levels of "peroxidases" which are excreted into the broth. It was therefore of interest to determine whether such peroxidases are capable of biotransforming these dibenzylbutanolide precursors to the desired cyclic products. Under the following conditions: $0.67 \mathrm{~g} / \mathrm{L}$ of 14 in a phosphate buffer $(\mathrm{pH} 6.3)$ containing 50 units of peroxidases derived from a 10 day old culture broth, 1 equivalent of hydrogen 
peroxide and at a temperature of $14^{\circ} \mathrm{C}$, an $87 \%$ yield of 15 was obtained in a 40 min incubation time. Although conditions have not been optimized, it is clear that this cell line provides a highly attractive route for gram scale production of podophyllotoxins.

\section{Section 3. Use of Plant Cell Cultures to Produce Higher Levels of Pharmaceutically Interesting Compounds.}

Tripterygium wilfordii Hook, commonly called Lei Gong Teng (Thunder God vine) or Mang Cao (rank grass) in China, is a perennial twining vine which has been used in herbal medicine in that country for several centuries. A refined extract from the root xylem of this plant, available in Chinese markets as tablets, has been used for the treatment of rheumatoid arthritis, various skin disorders, and more recently as a potential male contraceptive agent (19). Furthermore, the cytotoxic effects of two of the minor plant constituents, tripdiolide $(16, R=O H)$, and triptolide $(16, R=H)$, have also been noted (20). These data stimulated studies in our laboratory to develop cell cultures of $T$. wilfordii in an effort to obtain higher yields of these natural products. In particular, we hoped to ascertain which chemical structural types present in the tablets used in China, were responsible for the various pharmacological properties noted above. Some of our studies have been published (21-23) so only a brief summary of the most salient features are presented. The cell line of $T$. wilfordii, coded as TRP4a, produces a variety of diterpenes and triterpenes, and several representatives are shown in Fig. 6. A study of various

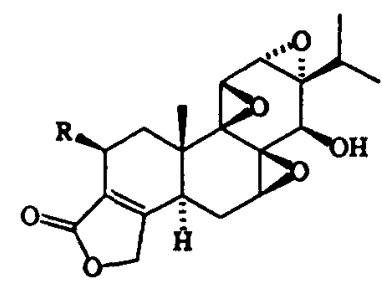

16

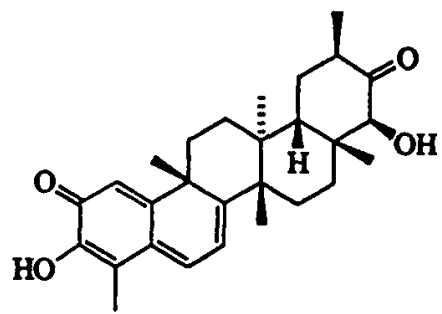

17

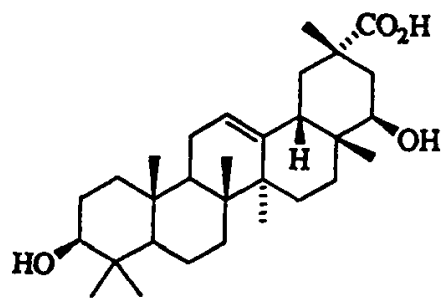

18

Fig. 6. Some representative structures of metabolites produced by the cell line of $T$. wilfordii.

growth parameters allowed the production of the diterpene triepoxides (16) at a level 40 times that in the growing plant (21) while elicitor stimulation significantly increased triterpene production (23). These experiments clearly indicate that higher levels of secondary metabolite production can be achieved in plant cell cultures when compared to the living plant.

\section{Section 4. Use of Plant Cell Cultures to Separate Pharmacological Activities in Complex Herbal Medicine Extracts.}

As noted above, $T$. wilfordii plant extracts have revealed interesting pharmacological properties in the treatment of various diseases in man. The question as to which components in the complex herbal mixture are responsible for these activities can be only answered when pure components are isolated and their pharmacology evaluated. The obvious advantages of plant cell culture versus living plants in terms of providing higher yields, a less complex and more easily handled mixture of metabolites afford an attractive route to separate and assess pharmacological properties of such herbal mixtures. Indeed studies with our TRP4a cell line, the separation of pure components and subsequent evaluation have shed some light on the properties of $T$. wilfordii derived herbal mixtures. A brief summary is presented here.

\section{(i) Immunosuppressive activity}

In developing drugs of potential interest in the treatment of rheumatoid arthritis, immunosuppression is considered as a significant parameter. For this reason, various metabolites from the TRP4a cell line were submitted for such assays, for example, lymphocyte proliferation, and in comparison with cyclosporine. The diterpene triepoxides, tripdiolide $(16, \mathrm{R}=\mathrm{OH})$ and triptolide $(16, \mathrm{R}$ $=\mathrm{H})$, were highly active $(95-99 \%$ immunosuppression at 100 picograms $/ \mathrm{ml})$ and further studies are clearly required to establish structure-activity relationships. 
(ii) Cytotoxic activity

In in vitro tests conducted recently against a spectrum of human cancer cell lines, the abovenoted triepoxides have revealed significant growth inhibition at nanogram levels and their potential in cancer chemotherapy is of interest.

(iii) Male contraceptive activity

Clinical data, available from extensive studies in China (19) have shown that tripdiolide and triptolide possess significant anti-spermatogenic activity in human males. Triptolide chlorohydrin, tripchlorolide, is presently in clinical trials.

(iv) Anti-inflammatory activity

On the other hand, the triterpene family of compounds isolated from the TRP4a cell line (structure 18, Fig. 6) possess anti-inflammatory activity and may well be responsible for the reason that T. wilfordii derived herbal extracts are useful in treatment of dermatological disorders. Extensive studies by Shibata et al (24) detail the pharmacological properties of these types of compounds.

\section{Conclusion}

The above discussion has revealed that the interplay between plant cell culture methodology and synthetic chemistry provides a unique opportunity for entertaining areas of interest related to a broad spectrum of scientists. The results obtained afford information of interest to synthetic and medicinal chemists, pharmacologists and enzymologists and finally scientists involved in the commercial production and development of drugs.

\section{Acknowledgement}

The author gratedfully acknowledges financial support from the Natural Sciences and Engineering Research Council of Canada. He is also grateful to Professor George R. Pettit and his collegues at the Arizona Cancer Institute, Tempe, Arizona, for cytotoxicity studies of our compounds.

\section{References}

1. J. P. Kutney. Synlett 1, 11 (1991), and references cited therein.

2. J. P. Kutney. Nat. Prod. Rep. 7, 85 (1990), and references cited therein.

3. J. P. Kutney. Acc. Chem. Res. 26, 559 (1993).

4. J. P. Kutney et al. Heterocycles 27, 621 (1988).

5. J. P. Kutney et al. Helv. Chim. Acta 59, 2858 (1976).

6. N. Langlois et al. J. Am. Chem. Soc. 98, 7017 (1976).

7. J. P. Kutney et al. Heterocycles 27, 1845 (1988).

8. J. P. Kutney et al. Can. J. Chem. 70, 2115 (1992).

9. J. P. Kutney et al. Heterocycles 36, 13 (1993).

10. J. Arnarp et al. Acta Chem. Scand. 47, 683 (1993).

11. J. Arnarp et al. Acta Chem. Scand. 47, 689 (1993).

12. J. Arnarp et al. Acta Chem. Scand. 47, 793 (1993).

13. D. C. Ayres and J. D. Loike, Lignans - Chemical, Biological and Clinical Properties, Cambridge University Press, Cambridge, 1990.

14. R. S. Ward. Synlett 8, 719 (1992), and references cited therein.

15. B. F. Issell et al. Etoposide (VP-16) - Current Status and New Developments, Academic Press, Orlando, 1984.

16. I. Jardine, Anticancer Agents Based on Natural Product Models, p. 319, Academic Press, New York, 1980.

17. A. J. Broomhead et al. Phytochemistry 30, 1489 (1991), and earlier references cited therein.

18. J. P. Kutney et al. Heterocycles 32, 2305 (1991).

19. S. Z. Qian et al. Contraception 51, 121 (1995).

20. S. M. Kupchan et al. J. Am. Chem. Soc. 94, 7194 (1972).

21. J. P. Kutney et al. Planta Medica 48, 158 (1983).

22. J. P. Kutney et al. Can. J. Chem. 70, 1455 (1992).

23. J. P. Kutney et al. Plant Cell Rep. 12, 356 (1993).

24. S. Shibata et al. Chem. Pharm. Bull. Japan 35, 3888 (1987). 